\title{
LncRNA GAS5 Regulates Osteosarcoma Cell Proliferation, Migration, and Invasion by Regulating RHOB via Sponging miR-663a
}

This article was published in the following Dove Press journal: Cancer Management and Research

\author{
Xinyu Yao \\ Xianan Li \\ Yi Luo \\ Xuezheng $\mathrm{Xu}$ \\ Jianfan Liu \\ Jie $\mathrm{Bu}$
}

Department of Orthopaedics, Hunan Cancer Hospital and The Affiliated Cancer Hospital of Xiangya School of Medicine, Central South University, Changsha, Hunan 4I00I3, People's Republic of China
Correspondence: Jie Bu

Email bujie_073I@yeah.net
Introduction: Emerging evidence has revealed the importance of long non-coding RNAs (lncRNAs) in carcinogenesis. The aim of this work was to investigate the roles of lncRNA growth arrest specific 5 (GAS5) in osteosarcoma (OS) progression.

Methods: Real-time quantitative polymerase chain reaction (RT-qPCR) was performed to explore GAS5, microRNA-663a (miR-663a), and ras homolog family member B (RHOB) expression levels in OS tissues and cells. Moreover, cell counting kit-8 assay, wound-healing assay, and transwell invasion assay were conducted to investigate biological roles of GAS5 in OS progression. In addition, mechanisms underlying the functions of GAS5 in OS were investigated by bioinformatic analysis, luciferase activity reporter assay, and rescue experiments.

Results: The GAS5 expression level was significantly decreased in OS tissues and cells compared with normal tissues and cells, and could negatively regulate miR-663a expression. Moreover, we found RHOB expression can be negatively regulated by miR-663a. Overexpression of GAS5 and RHOB suppresses, while overexpression of miR-663a stimulates, OS cell proliferation, migration, and invasion in vitro. In summary, we revealed lncRNA GAS5 was a downregulated lncRNA in OS and impaired OS malignant behaviors. In addition, this regulation relied on miR-663a and its target gene, RHOB.

Discussion: To sum up, we showed lncRNA GAS5 regulates OS progression via regulating the miR-663a/RHOB axis.

Keywords: lncRNA, GAS5, miR-663a, RHOB, osteosarcoma

\section{Introduction}

Osteosarcoma (OS) is a major bone cancer type and mainly affects children and adolescents. ${ }^{1,2}$ Control of OS remains a huge challenge and thus it is necessary to identify novel treatment markers for OS.

Long non-coding RNA (lncRNA), a type of non-coding RNA, is reported to play crucial roles in regulating carcinogenesis. ${ }^{3,4}$ However, only a small part of them have been functionally characterized. ${ }^{4}$ One of the main mechanisms of lncRNA in regulating tumorigenesis is that it can serve as a sponge for microRNA (miRNA) to regulate messenger RNA expression. ${ }^{5}$

LncRNA growth arrest specific 5 (GAS5), located at chromosome 1q25.1, is reported to play a tumor suppressive role in several cancers. ${ }^{6,7}$ For example, GAS5 was reported to inhibit colorectal cancer progression in vitro and in vivo through stimulating YAP degradation via ubiquitin modification. ${ }^{8}$ Moreover, low GAS5 
expression was found negatively correlated with overall survival of epithelial ovarian cancer patients. ${ }^{9}$ Mechanisms analyses indicated that GAS5 regulates PARP1 expression to affect the response of epithelial ovarian cancer to cisplatin. ${ }^{9}$

miR-663a was reported to have both tumor suppressive and oncogenic roles in cancers. ${ }^{10,11}$ For instance, miR663a was found to inhibit hepatocellular carcinoma progression via targeting transforming growth factor beta $1 .{ }^{10}$ On the contrary, miR-663a was found to have increased expression in renal cell carcinoma and be a predictor for shorter overall survival. ${ }^{11}$

A previous study showed GAS5 had reduced expression in OS cells, and inhibited cell migration and invasion through regulating miR-203a. ${ }^{12}$ However, detailed mechanisms related to the roles of GAS5 in OS remain to be explored. Therefore, this study was performed to investigate the acting mechanisms of GAS5 in OS.

\section{Materials and Methods}

\section{Human Tissues}

OS tissues $(n=20)$ and paired normal tissues $(n=20)$ were collected at Hunan Cancer Hospital and The Affiliated Cancer Hospital of Xiangya School of Medicine, Central South University. Written informed consent were obtained from all these 20 enrolled patients. The study protocol was approved by the ethics committee of Hunan Cancer Hospital and The Affiliated Cancer Hospital of Xiangya School of Medicine, Central South University and performed according to the Declaration of Helsinki.

\section{Cell Culture}

Two OS cells (MG63 and LM7) and mesenchymal stem cells (MSC) were purchased from Cell Bank of Chinese Academy of Sciences (Shanghai, China). OS cells were incubated in Roswell Park Memorial Institute-1640 (RPMI-1640; Thermo Fisher Scientific, Waltham, MA, USA) in supplement with $10 \%$ fetal bovine serum (FBS; Invitrogen, Thermo Fisher Scientific). MSC were incubated in hMSC serum-free medium (SCSP-661; Cell Bank of Chinese Academy of Science). The incubator was maintained at $37{ }^{\circ} \mathrm{C}$ and filled with $5 \% \mathrm{CO}_{2}$.

\section{Cell Transfection}

Sequences of GAS5 or RHOB were respectively inserted into pcDNA3.1 by GenScript (Nanjing,
Jiangsu, China) to construct expression vectors. Small interfering RNA against GAS5 (si-GAS5) and corresponding control (si-NC) were bought from GeneCopoeia (Guangzhou, Guangdong, China). miR663a mimic and corresponding control (NC-mimic) were also purchased from GeneCopoeia. Cell transfection for siRNAs, miRNAs, and expression vectors was conducted using Lipofectamine 2000 (Invitrogen) according to the supplier's instructions.

\section{Real-Time Quantitative Polymerase Chain Reaction (RT-qPCR)}

TRIzol (Invitrogen) was used to extract total RNA from tissues and cells based on the manufacturer's instructions. The PrimeScript RT Kit (Takara, Dalian, Liaoning, China) was used to synthesize complementary DNA from the extracted RNA sample. RT-qPCR using SYBR Green (Takara) was performed with the ABI 7500 system (Applied Biosystem, Foster City, CA, USA) to calculate relative expression levels of GAS5, miR-663a, and RHOB. GAPDH was employed as internal control for normalization of the expression of GAS5 and RHOB, while U6 snRNA was for miR-663a. Primer sequences used were as follows: GAS5: F (5'-CCTGTGAGGTATGGTGCTGG-3'), R (5'-CTGTGTGCCAATGGCTTGAG-3'); RHOB: F (5'-C TGCTGATCGTGTTCAGTAAGG-3'), R (5'-TCAATGTC GGCCACATAGTTC-3'); GAPDH: F (5'-GCACCGTCA AGGCTGAGAAC-3'), R (5'-TGGTGAAGACGCCAGTG GA-3'); miR-663a: F (5'-CTCGCTTCGGCAGCACA-3'), R (5'-AACGCTTCACGAATTTGCGT-3'); U6 snRNA: F (5'-GCTTCGGCAGCACATATACTAAAAT-3'), R (5'-C GCTTCACGAATTTGCGTGTCAT-3').

\section{Western Blot}

RIPA lysis buffer containing protease inhibitor (Beyotime) was used to extract total protein samples from tissues and cells. The BCA kit (Beyotime) was used to quantify the protein concentration according to the manufacturer's protocols. Then, the protein sample was separated with $10 \%$ SDS-PAGE and wet-transferred to PVDF membrane. After blocking with fat-free milk, the membrane was incubated with primary antibodies (anti-RHOB: ab53743, antiGAPDH: ab8245; Abcam, Cambridge, MA, USA) and secondary antibody (ab97040; Abcam). Finally, the BeyoECL kit (Beyotime) was employed to visualize protein signals. 


\section{Luciferase Activity Reporter Assay}

ENCORI (http://starbase.sysu.edu.cn/agoClipRNA.php? source=lncRNA) was employed to predict miRNA targets of GAS5, and we found miR-663a was a putative target. Messenger RNA target of miR-663a was also predicted using ENCORI, and we found RHOB was a highly potential target. Wild-type and mutant sequences of GAS5 or RHOB synthesized by GenScript (Nanjing, China) were inserted into pGL3 (Promega, Madison, WI, USA) to generate $\mathrm{wt} / \mathrm{mt}$ GAS5/RHOB luciferase vectors. Cells were co-transfected with luciferase vectors and synthetic miRNAs using Lipofectamine 2000 according to the manufacturer's instructions. Relative luciferase activities were measured with the Dual-Luciferase Reporter Assay System (Promega) after $48 \mathrm{~h}$ of transfection using Renilla luciferase activity as an internal control. Experiments were repeated in triplicate.

\section{Cell Proliferation Assay}

MTT assay was performed to detect cell viability. In brief, cells were seeded into a 96-well plate at the density of $2 \times 10^{4}$ cells/well and incubated at the above-described conditions for $24 \mathrm{~h}$. Then, cells were treated with $20 \mu \mathrm{L}$ MTT and $150 \mu \mathrm{L}$ dimethyl sulfoxide (DMSO) reagent at indicated time points. Finally, the optical density was read at $490 \mathrm{~nm}$ using a microplate reader.

\section{Cell Migration Assay}

Cells were seeded into a 6 -well plate at the density of $5 \times 10^{5}$ cells/well and maintained to $100 \%$ confluence. A scratch at the cell monolayer was created with a pipette tip and cultured in serum-free medium. Cell images were captured at 0 and 48 $\mathrm{h}$ using the CX23 microscope (Olympus, Tokyo, Japan).

\section{Cell Invasion Assay}

A Transwell chamber (Corning, Corning, NY, USA) coated with Matrigel (BD Biosciences, Franklin Lakes, NJ, USA) was used to evaluate the cell invasion ability. Cells in serum-free medium were seeded in the upper well, while serum containing medium was filled into the lower chamber. After $48 \mathrm{~h}$, invaded cells were fixed by methanol and stained by crystal violet. Invaded cell numbers were counted under the microscope.

\section{Statistical Analysis}

GraphPad Prism 7.0 (GraphPad Software, San Diego, CA, USA) was used for statistical analysis. Data from at least three experiments were exhibited as mean \pm standard deviation. Student's $t$-test and one-way analysis of variance (ANOVA) with Tukey post hoc test was used to analyze differences in groups. $P$ value less than 0.05 was considered as statistical significance.

\section{Results}

\section{LncRNA GAS5 Expression Level in OS}

The GAS5 expression level in OS tissues and cells was detected by the RT-qPCR method. The GAS5 expression level was remarkedly decreased in OS tissues compared with normal tissues (Figure 1A). In addition, the GAS5 expression level was significantly reduced in OS cells MG63 and LM7 compared with MSC (Figure 1B).
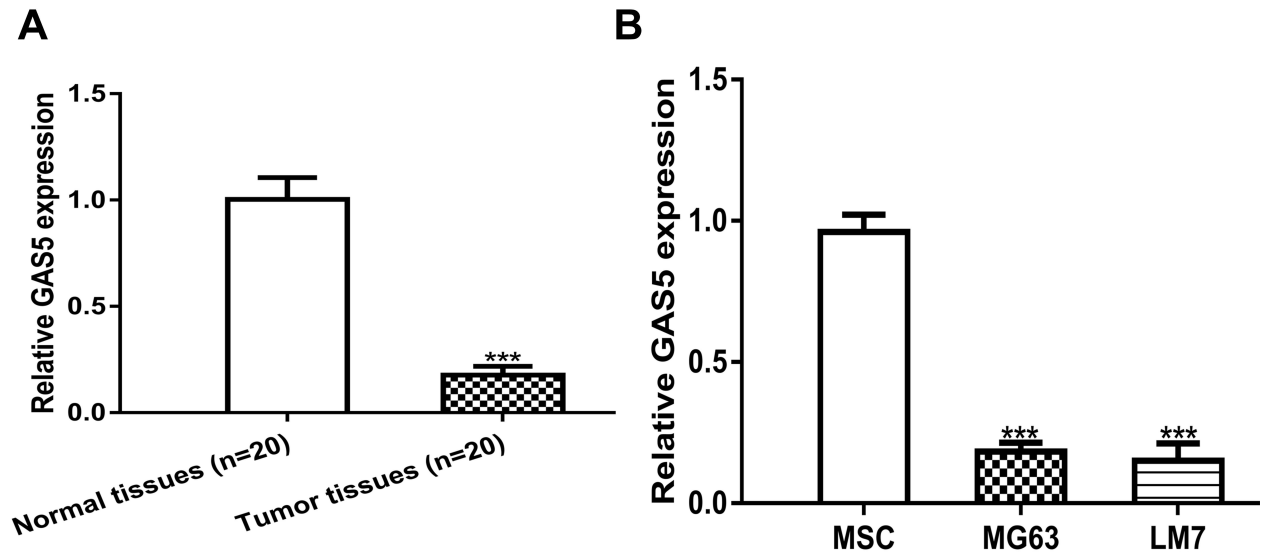

Figure I Decreased GAS5 expression in OS tissues and cells.

Notes: (A) GAS5 decreased expression in OS tissues $(n=20)$ compared with normal tissues $(n=20)$. (B) GAS5 decreased expression in OS cells (MG63 and LM7) compared with mesenchymal stem cells (MSC). $* * * P<0.001$.

Abbreviations: GAS5, growth arrest specific 5; MSC, mesenchymal stem cells; OS, osteosarcoma; RT-qPCR, real-time quantitative polymerase chain reaction. 


\section{GAS5 Knockdown Stimulates OS Cell} Proliferation, Migration, and Invasion

OS cells were transfected with si-GAD5, the transfection efficacy of which was detected by the RT-qPCR method (Figure 2A). MTT assay showed cell growth viability was stimulated via knockdown of GAS5 (Figure 2B). Moreover, OS cell migration and invasion abilities were significantly stimulated after the knockdown of GAS5 (Figure 2C and D).

A

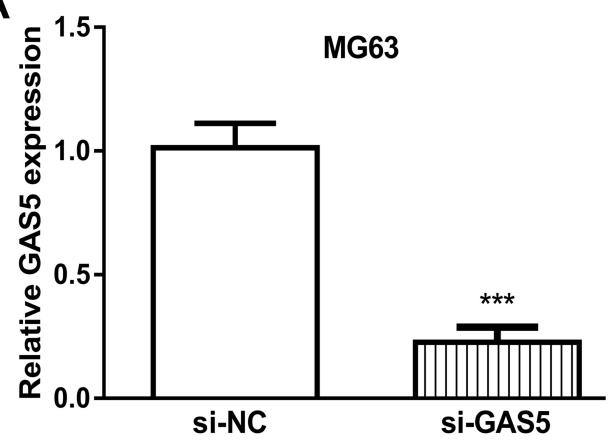

C

$0 \mathrm{~h}$

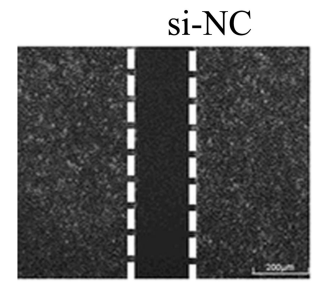

si-GAS5
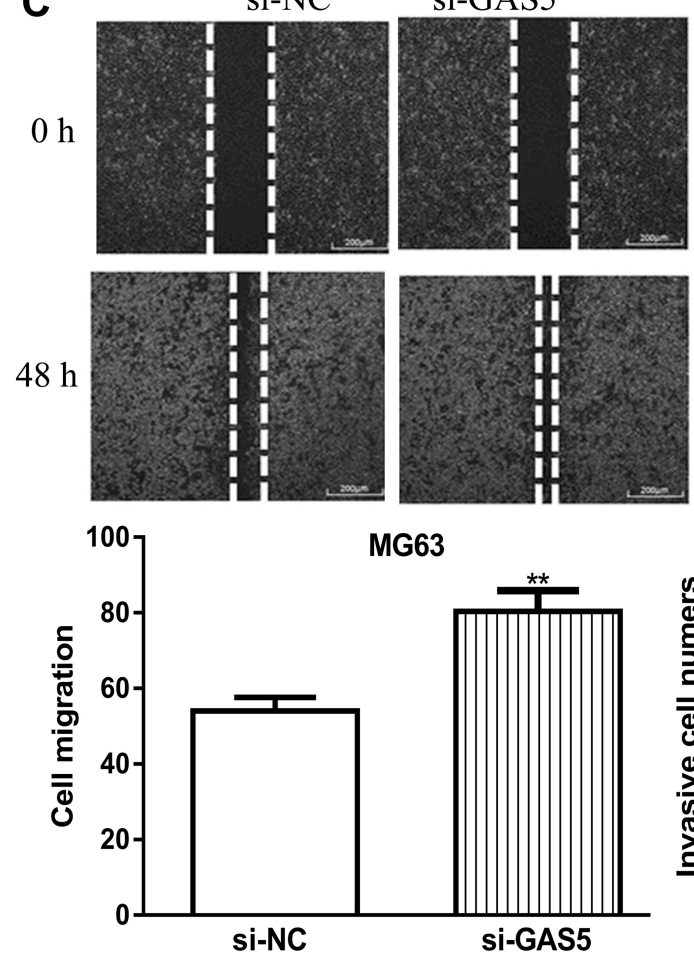

GAS5 Overexpression Inhibits OS Cell Proliferation, Migration, and Invasion

RT-qPCR assay showed pGAS5 transfection increases GAS5 expression levels in OS cells (Figure 3A). MTT assay showed OS cell growth ability was inhibited via GAS5 overexpression (Figure 3B). Wound-healing assay and transwell invasion assay indicated forced GAS5 expression suppresses OS cell migration and invasion (Figure $3 \mathrm{C}$ and D).

B

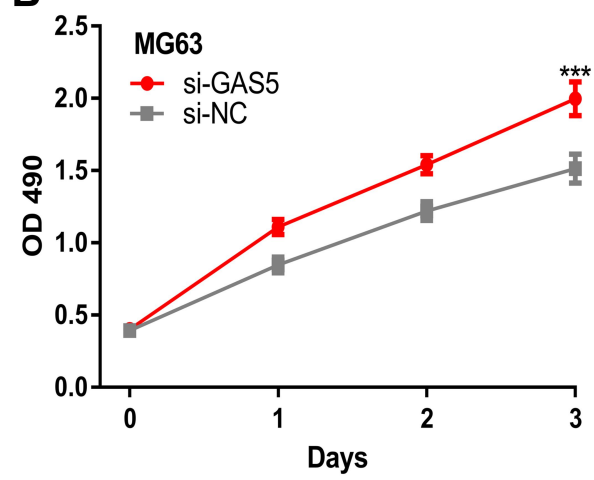

D

si-NC

si-GAS5

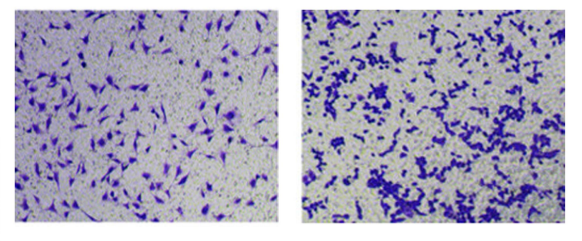

MG63

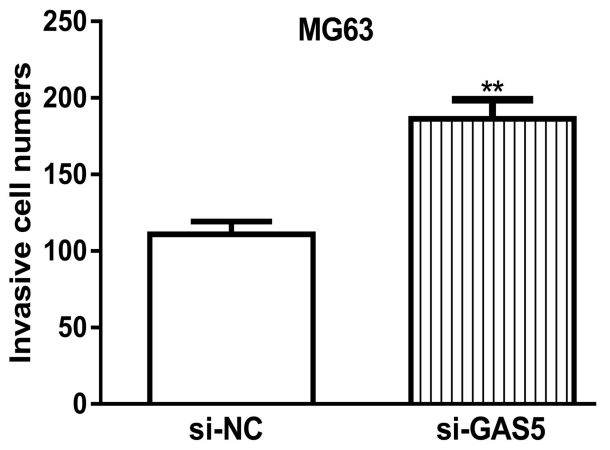

Figure 2 GAS5 knockdown promoted OS cell proliferation, migration, and invasion.

Notes: (A) GAS5 expression in OS cells transduced with si-GAS5 and si-NC. (B) Cell proliferation assay indicated that cell viability was stimulated after knockdown of GAS5. (C) Wound-healing assay showed the cell migration distance was increased by si-GAS5. (D) Transwell invasion assay revealed that cell invasion numbers in OS cells were enhanced by si-GAS5. $* * * P<0.001$, $* * p<0.01$.

Abbreviations: GAS5, growth arrest specific 5; OD, optical density; OS, osteosarcoma; si-GAS5, small interfering RNA against GAS5; si-NC, negative control small interfering RNA. 
A

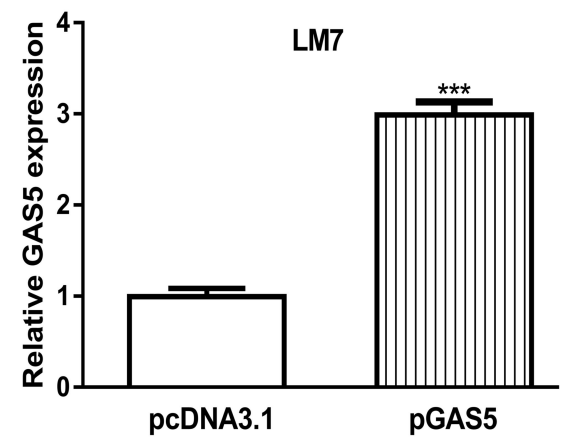

C pcDNA3.1

$0 \mathrm{~h}$
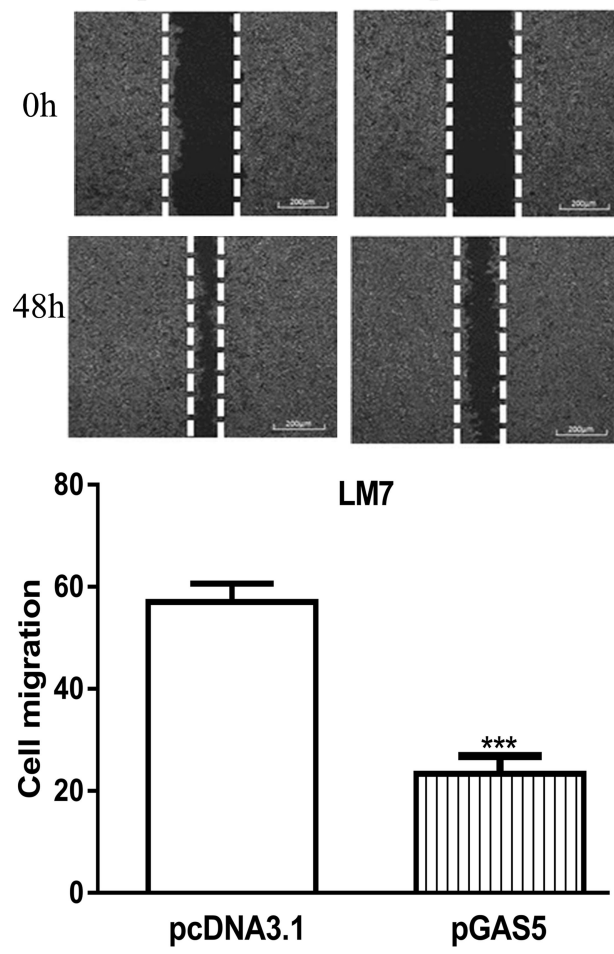

B

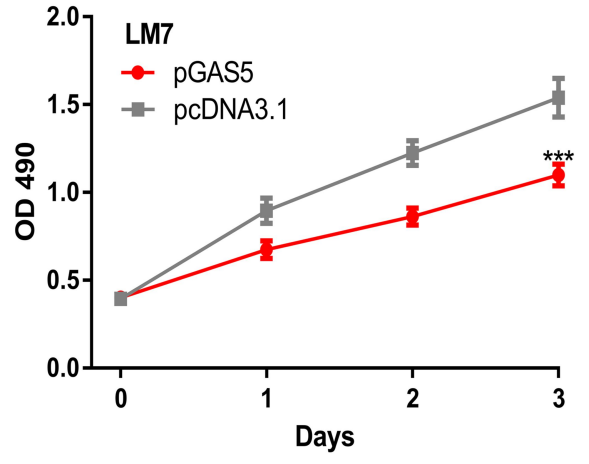

D pcDNA3.1 pGAS5
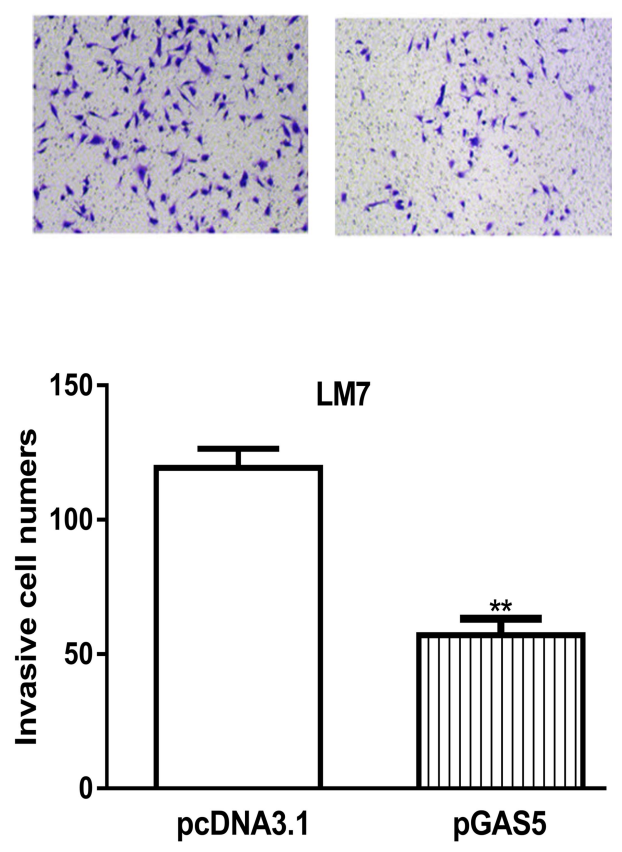

Figure 3 GAS5 overexpression inhibited OS cell proliferation, migration, and invasion.

Notes: (A) GAS5 expression in OS cells transduced with pGAS5 and pcDNA3.I. (B) Cell proliferation assay indicated that cell viability was inhibited after overexpression of GAS5. (C) Wound-healing assay showed the cell migration distance was repressed by pGAS5. (D) Transwell invasion assay revealed that cell invasion numbers in OS cells were reduced by pGAS5. $* * * p<0.001$, $* * p<0.01$.

Abbreviations: GAS5, growth arrest specific 5; OD, optical density; OS, osteosarcoma; pGAS5, pcDNA3.I contains the sequence of growth arrest specific 5.

\section{GAS5 Directly Interacts with miR-663a in OS}

ENCORI analysis indicated miR-663a was a possible target of GAS5 as a previous study suggested the potential of GAS5 and miR-663a (Figure 4A). ${ }^{13}$ The RT-qPCR method showed the miR-663a expression level was significantly upregulated in OS cells compared with normal cells (Figure 4B). The luciferase activity reporter assay showed relative luciferase activity was suppressed by miR-663a mimic in OS cells harboring a wt-GAS5 luciferase construct (Figure 4C). Moreover, miR-663a mimic or NC-mimic transfection did not alter the relative luciferase activity in OS cells transfected with mt-GAS5 luciferase construct (Figure 4C). In addition, GAS5 overexpression decreases miR-663a expression level in OS cells (Figure 4D).

\section{miR-663a Binds with RHOB in OS}

Furthermore, we showed RHOB was a target for miR-663a through ENCORI analysis (Figure 5A). RT-qPCR assay 


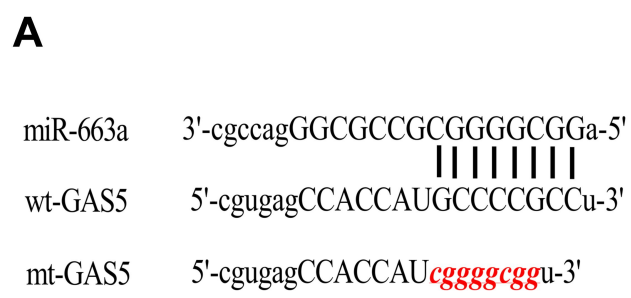

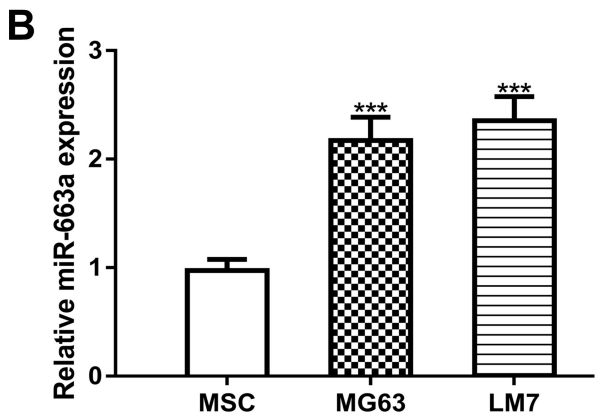
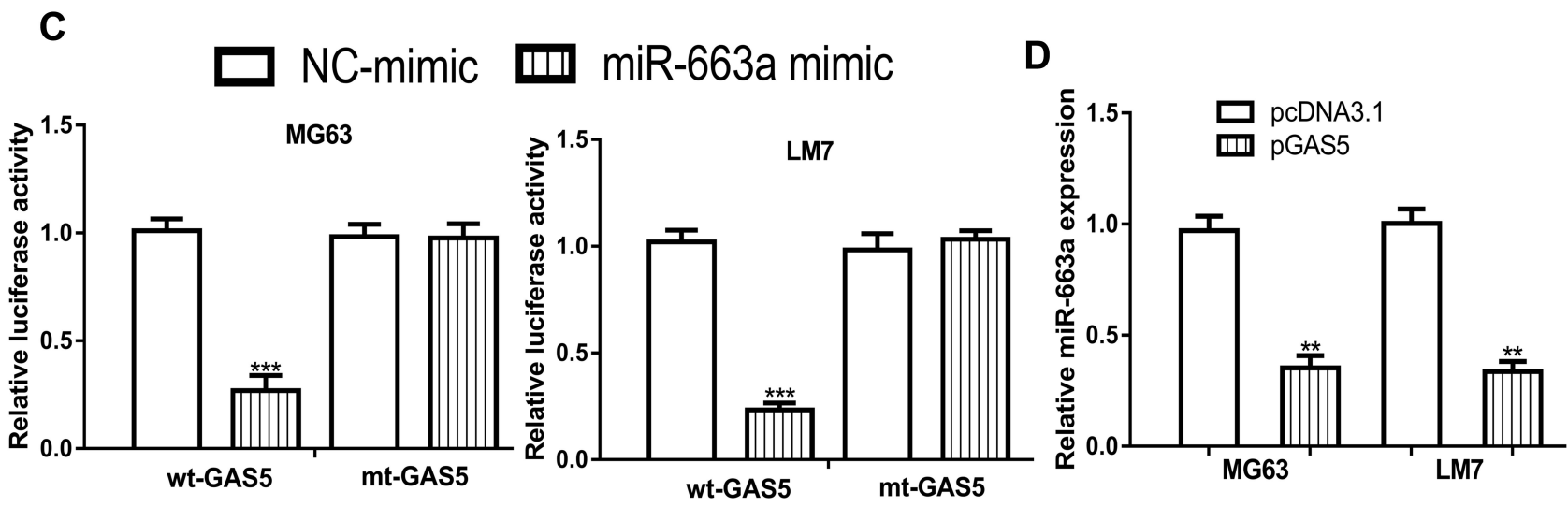

Figure 4 GAS5 directly interacts with miR-663a in OS cells.

Notes: (A) Interaction between GAS5 and miR-663a. (B) RT-qPCR showed miR-663a increased expression in OS cells (MG63 and LM7) compared with mesenchymal stem cells (MSC). (C) Luciferase activity reporter assay showed the relative luciferase activity was inhibited in OS cells transfected with wt-GAS5 luciferase vector and miR-663a mimic. (D) RT-qPCR showed miR-663a was decreased by pGAS5 in OS cells. ***P $<0.001$, **P $<0.01$.

Abbreviations: GAS5, growth arrest specific 5; miR-663a, microRNA-663a; MSC, mesenchymal stem cells; mt, mutant; NC-mimic, negative control for miR-663a mimic; OS, osteosarcoma; pGAS5, pcDNA3.I contains the sequence of growth arrest specific 5; RT-qPCR, real-time quantitative polymerase chain reaction; wt, wild-type.

indicated RHOB mRNA expression level was lower in OS cells than in normal cells (Figure 5B). Western blot assay results confirmed the results of the RT-qPCR assay (Figure 5C). The luciferase activity reporter assay showed miR663a mimic transfection inhibited the relative luciferase activity in OS cells harboring wt-RHOB (Figure 5D). Of note, the RT-qPCR assay showed miR-663a overexpression reduced the RHOB expression level in OS cells (Figure 5E). Western blot also showed miR-663a mimic transfection decreased the RHOB protein level in OS cells (Figure 5F). Besides that, we showed GAS5 overexpression increases both mRNA and protein levels of RHOB in OS cells (Figure 5G and $\mathrm{H}$ ).

\section{GAS5 Regulates OS Cell Proliferation, Migration, and Invasion via the miR-663a/ RHOB Axis}

Rescue experiments were performed to explore whether GAS5 regulates OS malignant behaviors via the miR663a/RHOB axis. We found miR-663a overexpression promotes, while RHOB overexpression inhibits, OS cell proliferation, migration, and invasion (Figure 6A-C). Importantly, we found miR-663a mimic transfection could partially abolish the inhibitory effects of pGAS5 and pRHOB on OS malignant behaviors (Figure 6A-C).

\section{Discussion}

Numerous lncRNAs have been reported to serve as oncogene or tumor suppressor genes in OS. For instance, $\mathrm{Wu}$ et al found a high LINC00324 level was an indicator for poorer overall survival of OS patients. ${ }^{14}$ Functional experiments showed LINC00324 could promote OS progression via stabilizing WD repeat-containing protein 66 expression. ${ }^{14}$ Another study showed non-coding RNA activated by DNA damage (NORAD) could bind with miR-199a-3p to stimulate OS malignant behaviors. ${ }^{15}$

Here, we showed lncRNA GAS5 decreased expression in OS tissues and cells compared with normal tissues and cells. We also investigated biological roles of GAS5 in OS via upregulating or downregulating its expression. We found GAS5 overexpression inhibits, while its knockdown promotes, OS malignant behaviors in vitro. Our results 
A
miR-663a
wt-RHOB
mt-RHOB
3'-cgecagggeGCCGCGGGGCGGa-5'
IIIIII
5'-aggcugggcCAGACUCCCGCCc-3'
5'-aggcugggcCAGACUgggcggc-3'

B

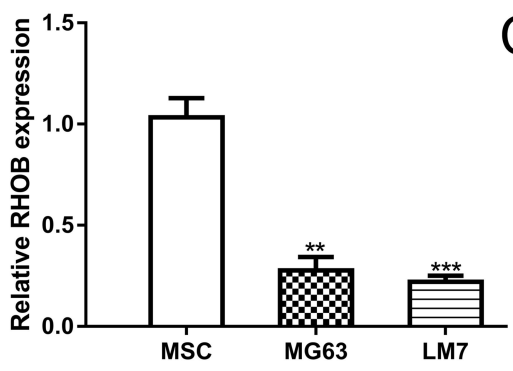

C

RHOB

GAPDH
LM7 MG63 MSC

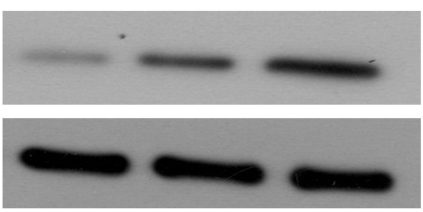

D

$\square$ NC-mimic $\boldsymbol{\square}$ miR-663a mimic
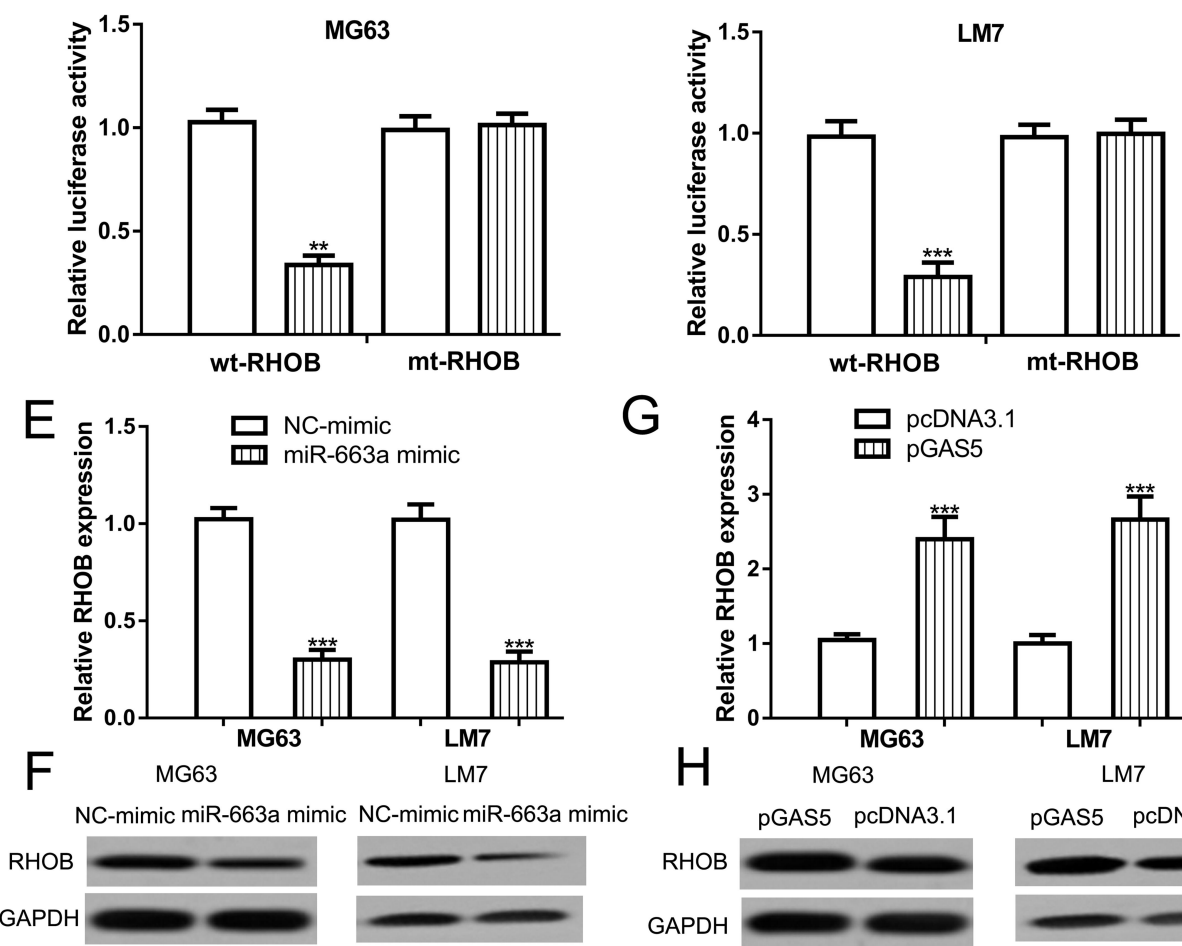

G

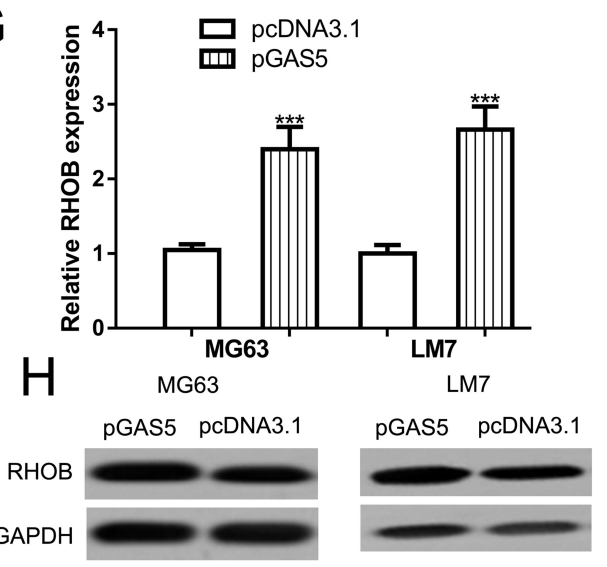

Figure 5 miR-663a directly interacts with RHOB in OS cells.

Notes: (A) Interaction between RHOB and miR-663a. (B) RT-qPCR showed RHOB decreased expression in OS cells (MG63 and LM7) compared with mesenchymal stem cells (MSC). (C) Western blot to detect RHOB expression in OS cells (MG63 and LM7) and mesenchymal stem cells (MSC). (D) Luciferase activity reporter assay showed relative luciferase activity was inhibited in OS cells transfected with wt-RHOB luciferase vector and miR-663a mimic. (E) RT-qPCR showed RHOB was decreased by miR663a mimic in OS cells. (F) Western blot showed RHOB protein level can be suppressed by miR-663a mimic. (G) RT-qPCR showed RHOB was increased by pGAS5 in OS cells. (H) Western blot indicated that RHOB protein level can be enhanced by PGAS5. $* * * P<0.001$, $* * P<0.01$.

Abbreviations: GAPDH, glyceraldehyde-3-phosphate dehydrogenase; miR-663a, microRNA-663a; MSC, mesenchymal stem cells; mt, mutant; NC-mimic, negative control for miR-663a mimic; OS, osteosarcoma; RHOB, ras homolog family member B; RT-qPCR, real-time quantitative polymerase chain reaction; wt, wild-type.

provided evidence that GAS5 plays a tumor suppressive role in OS progression, a role identified in colorectal cancer and epithelial ovarian cancer. ${ }^{8-10}$

It has been widely recognized that lncRNAs interact with miRNAs to regulate cancer progression. miR-663a was reported to have dual functions in cancers. ${ }^{10,11}$ Here, we showed the miR-663a expression level was increased in OS cells compared with normal cells. Moreover, interaction of GAS5 and miR-663a was validated by luciferase activity reporter assay. Furthermore, miR-663a overexpression was shown to stimulate OS cell malignant behaviors, indicating an oncogenic role of miR-663a. RHOB 

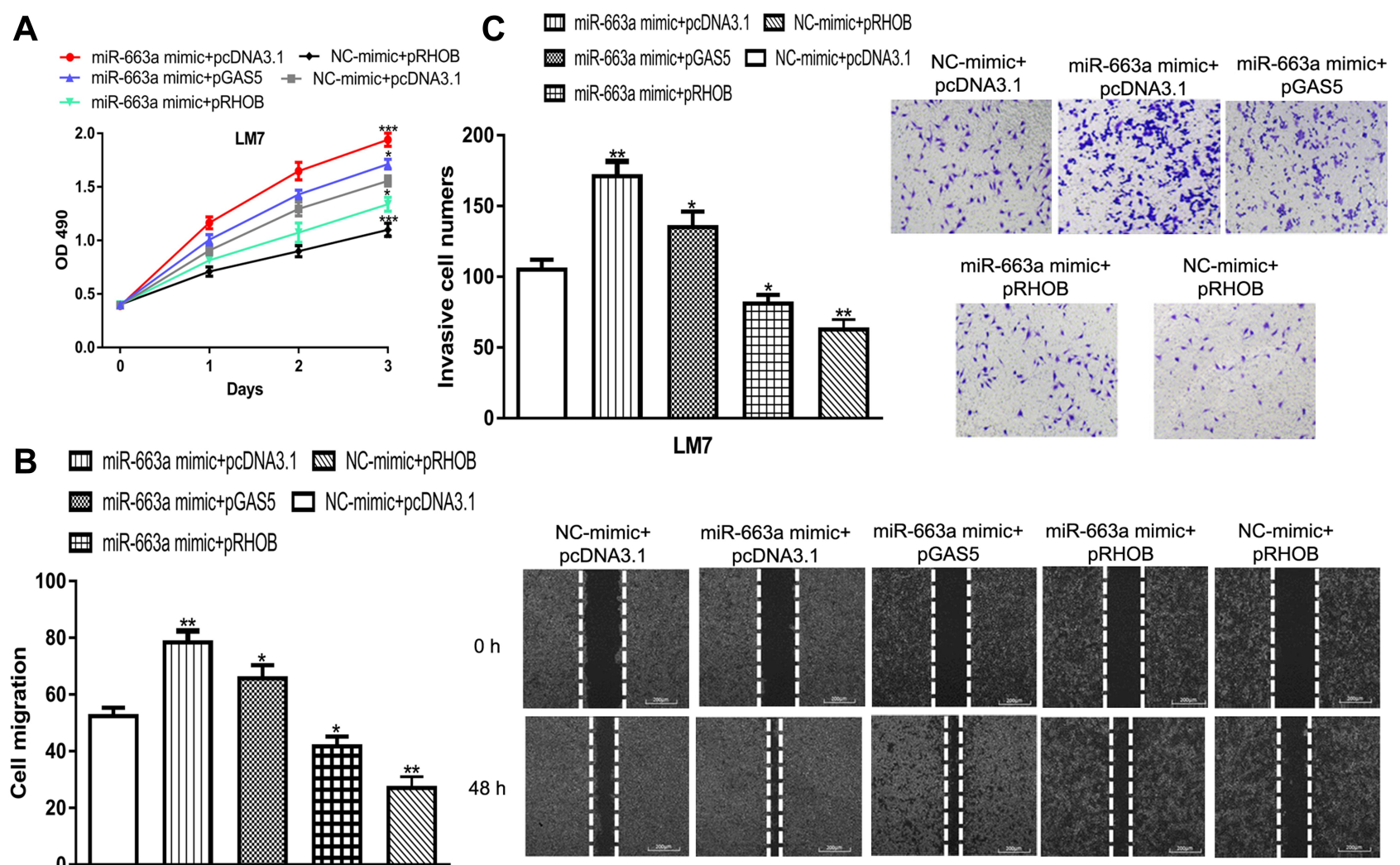

LM7

Figure 6 GAS5 regulates OS cell proliferation, migration, and invasion via regulating the miR-663a/RHOB axis.

Notes: (A) Cell proliferation assay indicated that cell viability was stimulated after overexpression of miR-663a, while inhibited after overexpression of RHOB. (B) Woundhealing assay showed the cell migration distance was increased by miR-663a mimic, while decreased by pRHOB. (C) Transwell invasion assay revealed that cell invasion numbers in OS cells was enhanced by miR-663a mimic, while reduced by $\mathrm{PRHOB}$. $* * * P<0.00 \mathrm{I}, * * P<0.0 \mathrm{I}, * P<0.05$.

Abbreviations: GAS5, growth arrest specific 5; miR-663a, microRNA-663a; NC-mimic, negative control for miR-663a mimic; OD, optical density; OS, osteosarcoma; PGAS5, pcDNA3.I contains the sequence of growth arrest specific 5; pRHOB, pcDNA3.I contains the coding sequence of ras homolog family member B; RHOB, ras homolog family member $B$.

was found to inhibit the epithelial to mesenchymal transition of bladder cancer cells. ${ }^{16}$ We found the RHOB expression level was decreased in OS by RT-qPCR and Western blot analyses, and its overexpression inhibits OS cell proliferation, migration, and invasion. Collectively, our results showed GAS5 regulates OS progression via the miR-663a/ RHOB axis.

\section{Conclusion}

In summary, we showed the GAS5 expression level was significantly reduced in OS, and could regulate OS cell proliferation, migration, and invasion through suppressing RHOB via sponging miR-663a.

\section{Author Contributions}

All authors made substantial contributions to conception and design, acquisition of data, or analysis and interpretation of data; took part in drafting the article or revising it critically for important intellectual content; gave final approval of the version to be published; and agree to be accountable for all aspects of the work.

\section{Disclosure}

The authors declare no conflicts of interest in this work.

\section{References}

1. Lin YH, Jewell BE, Gingold J, et al. Osteosarcoma: molecular pathogenesis and iPSC modeling. Trends Mol Med. 2017;23(8):737-755. doi:10.1016/j.molmed.2017.06.004

2. Cortini M, Avnet S, Baldini N. Mesenchymal stroma: role in osteosarcoma progression. Cancer Lett. 2017;405:90-99. doi:10.1016/j. canlet.2017.07.024

3. Mercer TR, Dinger ME, Mattick JS. Long non-coding RNAs: insights into functions. Nat Rev Genet. 2009;10(3):155-159.

4. Ponting CP, Oliver PL, Reik W. Evolution and functions of long noncoding RNAs. Cell. 2009;136(4):629-641. doi:10.1016/j. cell.2009.02.006 
5. Qi X, Zhang DH, Wu N, Xiao JH, Wang X, Ma W. ceRNA in cancer: possible functions and clinical implications. J Med Genet. 2015;52 (10):710-718. doi:10.1136/jmedgenet-2015-103334

6. Schneider C, King RM, Philipson L. Genes specifically expressed at growth arrest of mammalian cells. Cell. 1988;54(6):787-793. doi:10.1016/S0092-8674(88)91065-3

7. Yu Y, Hann SS. Novel tumor suppressor lncRNA growth arrest-specific 5 (GAS5) in human cancer. Onco Targets Ther 2019;12:8421-8436. doi:10.2147/OTT.S221305

8. Ni W, Yao S, Zhou Y, et al. Long noncoding RNA GAS5 inhibits progression of colorectal cancer by interacting with and triggering YAP phosphorylation and degradation and is negatively regulated by the m6A reader YTHDF3. Mol Cancer. 2019;18(1):143. doi:10.1186/ s12943-019-1079-y

9. Long $\mathrm{X}$, Song $\mathrm{K}, \mathrm{Hu} \mathrm{H}$, et al. Long non-coding RNA GAS5 inhibits DDP-resistance and tumor progression of epithelial ovarian cancer via GAS5-E2F4-PARP1-MAPK axis. $J$ Exp Clin Cancer Res. 2019;38(1):345. doi:10.1186/s13046-019-1329-2

10. Zhang C, Chen B, Jiao A, et al. miR-663a inhibits tumor growth and invasion by regulating TGF- $\beta 1$ in hepatocellular carcinoma. BMC Cancer. 2018;18(1):1179. doi:10.1186/s12885-018-5016-z
11. Zhou L, Pan X, Li Z, et al. Oncogenic miR-663a is associated with cellular function and poor prognosis in renal cell carcinoma. Biomed Pharmacother. 2018;105:1155-1163. doi:10.1016/j.biopha.2018.05.082

12. Wang Y, Kong D. LncRNA GAS5 represses osteosarcoma cells growth and metastasis via sponging MiR-203a. Cell Physiol Biochem. 2018:45(2):844-855. doi:10.1159/000487178

13. Zhang L, Wang Y, Zhang L, et al. ZBTB7A, a miR-663a target gene, protects osteosarcoma from endoplasmic reticulum stress-induced apoptosis by suppressing LncRNA GAS5 expression. Cancer Lett. 2019;448:105-116. doi:10.1016/j.canlet.2019.01.046

14. Wu S, Gu Z, Wu Y, Wu W, Mao B, Zhao S. LINC00324 accelerates the proliferation and migration of osteosarcoma through regulating WDR66. J Cell Physiol. 2020;235(1):339-348. doi:10.1002/jcp.28973

15. Wang X, Zou J, Chen $\mathrm{H}$, et al. Long noncoding RNA NORAD regulates cancer cell proliferation and migration in human osteosarcoma by endogenously competing with miR-199a-3p. IUBMB Life. 2019;71(10):1482-1491. doi:10.1002/iub.2064

16. Li Z, Li Y, Wang Y. miR-19a promotes invasion and epithelial to mesenchymal transition of bladder cancer cells by targeting RhoB. J BUON. 2019;24(2):797-804.

\section{Publish your work in this journal}

Cancer Management and Research is an international, peer-reviewed open access journal focusing on cancer research and the optimal use of preventative and integrated treatment interventions to achieve improved outcomes, enhanced survival and quality of life for the cancer patient.
The manuscript management system is completely online and includes a very quick and fair peer-review system, which is all easy to use. Visit http://www.dovepress.com/testimonials.php to read real quotes from published authors. 